\title{
Dietary restriction slightly affects glucose homeostasis and delays plasma cholesterol removal in rabbits with dietary lipid lowering
}

\begin{tabular}{|c|c|}
\hline Journal: & Applied Physiology, Nutrition, and Metabolism \\
\hline Manuscript ID & apnm-2017-0876.R1 \\
\hline Manuscript Type: & Article \\
\hline Date Submitted by the Author: & 22-Mar-2018 \\
\hline Complete List of Authors: & $\begin{array}{l}\text { Yu, Qi; Xi'an Medical University, Shaanxi Key Laboratory of Ischemic } \\
\text { Cardiovascular Diseases \& Institute of Basic and Translational Medicine } \\
\text { Liu, Ruihan; Central Hospital Affiliated to Zhengzhou University, } \\
\text { Department of Pathology } \\
\text { Han, Lijuan ; Xi'an Medical University, Shaanxi Key Laboratory of Ischemic } \\
\text { Cardiovascular Diseases \& Institute of Basic and Translational Medicine } \\
\text { Zhang, Guangwei ; Xi'an Medical University, Shaanxi Key Laboratory of } \\
\text { Ischemic Cardiovascular Diseases \& Institute of Basic and Translational } \\
\text { Medicine } \\
\text { Guan, Hua; Xi'an Medical University, Shaanxi Key Laboratory of Ischemic } \\
\text { Cardiovascular Diseases \& Institute of Basic and Translational Medicine } \\
\text { Pan, Qi; Xi'an Medical University, Shaanxi Key Laboratory of Ischemic } \\
\text { Cardiovascular Diseases \& Institute of Basic and Translational Medicine } \\
\text { Wang , Siwang ; The Fourth Military Medical University, Institute of } \\
\text { Material Medical, School of Pharmacy } \\
\text { Liu, Enqi; Xi'an Medical University, Shaanxi Key Laboratory of Ischemic } \\
\text { Cardiovascular Diseases \& Institute of Basic and Translational Medicine }\end{array}$ \\
\hline Keyword: & dietary restriction, lipid lowering, atherosclerosis, glucose homeostasis \\
\hline $\begin{array}{r}\text { Is the invited manuscript for } \\
\text { consideration in a Special } \\
\text { Issue? : }\end{array}$ & N/A \\
\hline
\end{tabular}


Dietary restriction slightly affects glucose homeostasis and delays plasma cholesterol removal in rabbits with dietary lipid lowering

Qi Yu ${ }^{1,2 \dagger *}$, Ruihan $\mathrm{Liu}^{3 \dagger}$, Lijuan $\mathrm{Han}^{1}$, Guangwei Zhang ${ }^{1}$, Hua Guan ${ }^{1}$, Qi Pan ${ }^{1}$, Siwang Wang ${ }^{2}$, Enqi $\mathrm{Liu}^{1}$

${ }^{1}$ Shaanxi Key Laboratory of Ischemic Cardiovascular Diseases \& Institute of Basic and Translational Medicine, Xi'an Medical University, Xi'an 710021, China;

${ }^{2}$ Institute of Material Medical, School of Pharmacy, The Fourth Military Medical University, Xi'an

710032, Xi'an 710021, China;

${ }^{3}$ Department of Pathology, Central Hospital Affiliated to Zhengzhou University, Zhengzhou 450007,

China;

Address correspondence to:

Dr. Qi Yu, Institute of Basic and Translational Medicine, Xi'an Medical University, Xi'an, 710021, China. E-mail: qiyu6028@hotmail.com 


\section{Abstract}

Dietary restriction (DR) has been reported to promote the beneficial effects on atherosclerotic progression, lipid and glucose metabolism, but little is known about these effects can be enhanced or weakened by dietary lipid lowering. After 12 weeks of the high-cholesterol diet (HCD) feeding, hypercholesterolemic rabbits were fed with either a chow diet ad libitum (AL) or a chow diet with DR for 16 weeks of dietary lipid lowering. Here, we found the DR group exhibited a loss in body weight, small internal organs and the reduced fat mass, but the AL group accumulated more subcutaneous fat than the baseline group. DR treatment slightly worsened glucose tolerance but enhanced insulin sensitivity, and a slight effect of DR on insulin secretion was also observed. After diet cholesterol withdrawal, rabbits showed persistently lowering of total cholesterol and triglyceride in plasma. The DR group had significantly higher plasma total cholesterol than the AL group at the most time points during 7 to 16 weeks of lipid lowering. Although both AL and DR groups developed more severe atherosclerosis than baseline group, DR did not improve atherosclerotic progression and the accumulation of macrophages and smooth muscle cells as well. We concluded that DR affected glucose and lipid metabolism but did not ameliorate atherosclerosis in rabbits when associated with lipid lowering by the dietary cholesterol withdrawal.

Key words: dietary restriction, lipid lowering, atherosclerosis, glucose homeostasis. 


\section{Introduction}

As a pathological basis of cardiovascular disease (CVD), atherosclerosis is characterized as a complex multi-factorial process that shows the accumulation of lipids and fibrous elements in arterial walls. Atherosclerosis could be worsened by behavioral risk factors such as cigarette smoking, poor quality diet, physical inactivity, excessive alcohol consumption and obesity. Conversely, healthy lifestyles were considered that could be beneficial for CVD (Millen et al. 2014). Dietary restriction (DR) has been found to extend life in various species. Although there is still controversy about the effect of DR on mammals, some studies have proven that DR can directly ameliorate atherosclerotic progression in rodents and human (Guo et al. 2002; Fontana et al. 2004; Hursting et al. 2010; Dolinsky and Dyck 2011). Interestingly, given a large number of patients who are subjected to lipid-lowering therapy, a question arises whether DR can promote the beneficial effects on blood lipid control and atherosclerotic regression. To address this question, we developed HCD-induced hypercholesterolemia in rabbits; then, dietary cholesterol withdrawal along with DR was employed to intervene these rabbits, and the effects of DR on atherosclerotic progression, lipid and glucose metabolism were observed.

\section{Materials and methods}

\section{Experimental design}

We designed this experiment as shown in Fig. 1. 50 Japanese white rabbits were fed by a high-cholesterol diet (HCD; containing 0.3\% cholesterol and 3\% corn oil; approximately 120-180 g per day) for 12 weeks to develop mild atherosclerosis (Yu et al. 2012b). HCD contains approximately 2.63 kilocalories per gram. Next, these rabbits were randomly divided into four groups, and the baseline was sacrificed immediately $(n=10)$. Another group was continually fed by HCD in another 16 weeks as a positive control group $(n=10)$. The rest of two groups were fed with a normal diet instead of a HCD. Among them, the DR group $(n=15)$ was fed by the dietary restriction and received $60 \%$ of the diet consumption in the group that was fed by ad libitum (AL, $\mathrm{n}=15$ ) (approximately $90 \mathrm{~g}$ and 150-180 $\mathrm{g}$ per day, respectively) (Guo et al. 2002). A normal diet is constituted by crude protein (18.5\%), crude fat $(2.7 \%)$, crude fiber $(13.4 \%)$, ash $(8.5 \%)$, carbohydrate $(42 \%)$ and others $(14.9 \%)$, which contains approximately 2.43 kilocalories per gram. The amount of food consumption of each rabbit was monitored by daily check and was calculated each week. This study was carried out according to the 
Guidelines for Animal Experimentation of Xi'an Medical University and the Guide for the Care and Use of Experimental Animals (Vol. 1, 2nd ed., 1993, and Vol. 2, 1984, available from the Canadian Council on Animal Care, 190 O'Connor St, Suite 800, Ottawa, ON K2P 2R3, Canada, or on their Web site at www.ccac.ca). All rabbits were provided by the Laboratory Animal Center of Xi'an Medical University, and the experimental protocols were approved by the Laboratory Animal Administration Committee of Xi'an Medical University (No. 2015XYJZS003).

\section{Assessment of plasma parameters}

Plasma total cholesterol (TC), triglyceride (TG), and high density lipoprotein-cholesterol (HDL-C) were measured weekly by using commercial assay kits (BioSino Bio-technology and Science Inc, Beijing, China). Plasma glucose was measured every four weeks by using a commercial assay kit (BioSino Bio-technology and Science Inc, Beijing, China). The measurement protocols of plasma TC, TG, HDL-C and glucose were described in our previous study (Yu et al. 2012a).

\section{Analysis of glucose metabolism}

Intravenous glucose tolerance tests (IGTT) and intravenous insulin tolerance test (IITT) were performed at the end of study as previously described (Cheng et al. 2015). Briefly, the rabbits were injected with glucose solution intravenously after $16 \mathrm{~h}$ of fasting, and then blood samples were drawn at $5,10,15,20,30,45,60,75$ and $120 \mathrm{~min}(2 \mathrm{~g} / \mathrm{kg}$ body weight). For IITT, after the intravenous injection of insulin (1 U/kg body weight; Wanbang Co., Jiangsu, China), blood samples were collected to examine blood sugar at 5, 10, 15, 30, 45, 60, 90 and 120 min, and plasma insulin was also measured at 0, 60 and 120 min by using an ELISA kit (Shibayagi Co., Gunma, Japan). The areas under carve (AUC) of IGTT and IITT were quantified according to previously described (Cheng et al. 2015). At 0, 8, 12 and 16 weeks of dietary lipid lowering, the plasma glucagon levels of the AL and DR groups were measured by an ELISA kit (Merck Millipore Co., Billerica, MA, USA).

\section{Histology and immunohistochemistry}

After formalin fixation, the whole aortas were stained with Sudan IV and photographed for the evaluation of the gross size of atherosclerotic lesions. For histological analysis, the aortic arch of each rabbit was cut into cross 8 to 10 sections $(4 \mu \mathrm{m})$ as previously described (Yu et al. 2014). All sections 
were stained with Hematoxylin and Eosin (H\&E) and Elastica van Gieson (EVG) and immunohistochemically stained with RAM11 antibody against macrophages (dilution 1:200; Dako Inc., CA, USA) and smooth muscle $\alpha$-actin (dilution 1:200; Thermo Fisher Scientific Inc., CA, USA). Adipose tissue and liver were made to sections and stained with H\&E, and adipocyte size was evaluated under a light microscope as previously described (Waqar et al. 2010). All sections (H\&E, EVG and immunostaining) were captured with a digital camera (Olympus Inc., Tokyo, Japan) and quantified by ImageJ as previously described (Zhang et al. 2015).

Western blotting

Total protein was extracted from plasma and western blotting was performed as previously described (Fukuda et al. 2017). The primary antibody was against adiponectin (R\&D systems; 1:1000). Western blotting analysis was performed as previously described, and relative protein expression was quantified by ImageJ (Gassmann et al. 2009).

\section{Statistical analysis}

All data are expressed as mean \pm SE. Comparisons for two groups were performed by t-test. Comparisons for multiple groups were conducted by one-way or two-way ANOVA with Bonferroni test. $\mathrm{P}<0.05$ is considered as a statistically significant.

\section{Results}

\section{Body weight, internal organs and fat mass}

In 16 weeks of diet cholesterol withdrawal, the AL and DR groups consumed $150 \pm 7.24 \mathrm{~g} /$ day or 89.35 $\pm 0.02 \mathrm{~g}$ /day of a chow diet, respectively. The DR group showed that body weights were remarkably reduced when compared with the AL group from 8 weeks to 16 weeks (Fig. 2A). Interestingly, the HCD-fed rabbits had the lower body weight than the AL or DR group (Fig. 2A). To study the weight of major internal organs, we found that both DR and HCD groups had dramatically reduced weights of liver when compared with the baseline and AL group, respectively (Fig. 2B).

We next analyzed the distribution of adipose tissue, finding that visceral and subcutaneous adipose tissues were significantly decreased in the DR group (Fig. 3A). Conversely, the AL group accumulated more subcutaneous fat, and the HCD group accumulated more visceral fat than the baseline group (Fig. 
3A). Moreover, we also analyzed the adipocytes size between the AL and DR groups, indicating that the diameter of visceral adipocytes in DR-treated rabbits were dramatically reduced in a narrow range of 20-40 $\mu \mathrm{m}$ (Fig. 3B). Considering that the small size of adipocytes may affect glucose and lipid metabolism via adipocytokines, we also measured plasma adiponectin to check the effect of DR on endocrine function of adipose tissue. As showed by a result from Western blotting, we did not find any difference between the AL and DR groups (Fig. 3C).

\section{DR affects glucose and insulin metabolism}

By comparing the levels of fasting plasma glucose, we found that there was no obvious difference between the AL and DR groups (Fig. 4A). We also performed IGTT and IITT to evaluate glucose intolerance and insulin sensitivity on these rabbits. We found the DR group had significantly increased blood glucose levels at last two time points in IGTT, but no difference in comparison of AUC values (Fig. 4B). However, after intravenous injection of insulin, IITT revealed that the DR group had significantly reduced blood glucose levels at last three time points, but no difference in comparison of AUC values (Fig. 4C).

To study the effect of DR on insulin and glucagon secretion, we measured the levels of these hormones by using a pair of commercial ELISA kits. Interestingly, DR-treated rabbits exhibited the lower plasma insulin than the AL group and reached statistical significance at $30 \mathrm{~min}$ after intravenous injection of glucose (Fig. 5A). However, we did not find any significant difference in plasma glucagon between two groups (Fig. 5B).

\section{DR affects dietary lipid lowering}

By feeding 16 weeks of a chow diet, a declining trend of plasma TC was found in both AL and DR groups (Fig. 6A). Notable, the DR group had relatively higher plasma TC than the AL group from 7 to 16 weeks of rabbits fed with a chow diet, and the statistical significances were achieved at the most time points during this period ( $\mathrm{p}<0.05$ or $\mathrm{p}<0.01$ versus the AL group; Fig. $6 \mathrm{~A}$ ). We also examined plasma HDL-C, whereas there was no difference between the AL and DR groups (Fig. 6B). Moreover, plasma TG levels in both AL and DR groups were also slowly declining during 16 weeks of diet cholesterol withdrawal, and DR-treated rabbits showed lower plasma TG in 2 and 3 weeks than the AL group (Fig. 6C). 


\section{DR does not ameliorate atherosclerotic progression}

To quantify of the gross lesion, The AL and DR groups showed severely atherosclerotic lesions at the end of this study $\left({ }^{* *} \mathrm{p}<0.01\right.$ and ${ }^{* * *} \mathrm{p}<0.001$ versus the baseline group, respectively; Fig. 7). In spite of the AL and DR-treated rabbits underwent 16 weeks of a chow diet feeding, their gross lesions still accounted for approximately $65 \%$ and $57 \%$ of the whole aorta (respectively), when contrasted to $72 \%$ of the whole aorta in the HCD group (Fig. 7). To explore the effect of DR on atherosclerotic progression, we also compared the gross lesions between the DR and AL groups, showing no significant difference (Fig. 7). Given that intimal components determine the inflammation and stability in atherosclerotic lesions, we next analyzed the effect of DR on intimal lesions, macrophages and SMCs. However, we did not find any difference when compared the AL group with the DL group (Fig. 8).

\section{Discussion}

In this study, we intended to elucidate the effect of DR on atherosclerotic progression, lipid and glucose metabolism in the rabbits with dietary lipid lowering. After 16 weeks of DR, body weight, internal organs and fat mass were dramatically reduced in these rabbits. In light of that visceral adipose tissues play a key role for the CVD, we investigated the distribution of adipose tissue and the size of visceral adipocyte in these rabbits (Muzumdar et al. 2008). Unlike the HCD-fed rabbits, an alternative normal diet can decrease visceral fat mass rather than subcutaneous fat mass (Yu et al. 2012c). Furthermore, the DR causes a decrease in visceral fat mass and the size of adipocytes, which be deemed as a beneficial factor for metabolic disease (Skurk et al. 2007). Especially for small size of adipocytes, it has been reported that improves atherosclerotic regression and lipid metabolism whereby the production of the adipocytokines (Laforest et al. 2015). Although the current study does not support that DR promote a significant increase of plasma adiponectin, there is a possible explanation that the function of small adipocytes is suppressed by hypercholesterolemia in these animals. Interestingly, the positive control group also had reduced body weight and small internal organs in another 16 weeks of HCD-fed, which was attributed to that a long-term of HCD intake may affect rabbits' metabolism as previously described (Yu et al. 2012b; Fan et al. 2015)

It has been reported that DR can improve glucose intolerance and insulin sensitivity from various 
animal models to human being (Wang et al. 2011; Roth and Polotsky 2012; Pires et al. 2014). However, when rabbits with dietary lipid lowering, a pair of paradoxical results indicated that DR slightly worsened glucose intolerance but improved insulin sensitivity. Nonetheless, in light of a large fluctuation in plasma glucose levels of rabbits, the slight changes in the results of IGTT and IITT do not mean DR contributing to significant effect on glucose metabolism. In clinical practice, the effect of DR on glucose metabolism should be carefully evaluated. Considering that DR might suppress the secretion of insulin, we used a commercial ELISA kit to check this effect, indicating that DR indeed promoted a slight reduction in plasma insulin levels after stimulated by glucose intravenous injection. Under the hypoglycemic condition, pancreatic $\alpha$ cells produce glucagon to inhibit $\beta$ cells for insulin secreting, and glucagon is deemed as an antagonist against the physiological effects of insulin as well (Koubova and Guarente 2003). Accordingly, we measured the levels of plasma glucagon by another commercial ELISA kit. Despites no obvious difference in plasma glucagon, the inhibition of DR on insulin levels via paracrine suppression by glucagon still need to be further investigated.

After removed cholesterol from the diet, hypercholesterolemic rabbits presented very slowly declining in the plasma lipids during this period, which was ascribed to that excess cholesterol accumulation in extravascular site and later diffused into the blood (Khanna et al. 2013). Of note, DR did not enhance the clearance of plasma TC. Inversely, a retardant decline of plasma TC levels in the DR-treated rabbits was observed. A previous study revealed that dietary restriction gave rise to significantly high TC in the hypercholesterolemic rabbits, and the mechanism was suggested that dietary restriction might cause inhibition of hepatic uptake, an increased production of lipoprotein, increased intestinal absorption of cholesterol and the activity of $\mathrm{N}$-acetyl- $\beta$-glucosaminidase (Lacombe et al. 1983). Contrasted to this study, we further observed that DR increased plasma TC after dietary cholesterol withdrawal. Moreover, DR also not increased HDL-C when compared with rabbits with AL fed, which might be attributed to that DR promoted overproduction of LDL-C and VLDL-C rather than HDL-C as previously described (Lacombe et al. 1983). Another interesting question is whether DR can affect the function of HDL or cholesterol ester transport protein (CETP). A human dietary intervention study has revealed that a 16 weeks of very low calorie diet feeding can decrease plasma CETP concentration, but not affect HDL-C and the function of HDL (Wang et al. 2011). 
After 12 weeks of high-cholesterol diet feeding, rabbits showed obvious gross lesions of atherosclerosis ( $26 \%$ of the whole aorta) as the early- and middle-stage of atherosclerotic lesions in human (Yu et al. 2012b; Fan et al. 2015). Interestingly, despite that the AL and DR-treated rabbits underwent another 16 weeks of diet cholesterol withdrawal, they still developed more severe atherosclerosis than the baseline group. Based on this finding, we suggest that the term of HCD-fed could be shorten when rabbit model is used for the study of atherosclerosis or hypercholesterolemia. Moreover, whereby various comparisons in the gross and intimal lesions, the accumulation of macrophages and SMCs in the DR and AL groups, our data do not support that DR can improve atherosclerotic progression. Considering that humans and rabbits are LDL-enriched mammals and their lipoprotein profile are more similar than mice and rats, the current study may provide a novel insight into the effect of DR on cardiovascular system (Fan et al. 2015). Moreover, rabbits have greater body size than rodents, facilitating to be evaluated by metabolic indexes such as food consumption, body weight and plasma parameters. Therefore, the current study also provides valuable information that DR may promote the adverse effect on the cholesterol metabolism, which may finally retard the regression of atherosclerosis when patients with dramatic lipid lowering. Indeed, there were two primate studies also presented the opposite results: The University of Wisconsin group showed that the DR monkeys had a 50\% reduction in cardiovascular disease versus controls, whereas the Wake Forest University group showed that DR group had no improvement in cholesterol, blood pressure, or coronary artery atherosclerosis (Cefalu et al. 2004; Colman et al. 2009). Combined to these results, the current study suggests that the benefits of DR may need to be further investigated in the patients with hyperlipidemia or the CVD.

Taken together, the current study has proven that DR can cause the dysfunction of cholesterol metabolism in rabbits with dietary lipid lowering, which may further neutralize the benefits of DR on the CVD. In light of fact that cholesterol and glucose homeostasis are maintained by extremely sophisticated manners in mammals, we conclude that simple DR does not ameliorate atherosclerotic regression in rabbits when associated with lipid lowering by the dietary cholesterol withdrawal.

\section{List of abbreviations}

CR, calories restriction; HCD, high-cholesterol diet; AL, ad libitum; TC, total cholesterol; TG, triglyceride; HDL-C, high-density lipoprotein cholesterol; SMCs, smooth muscle cells; CVD, cardiovascular disease; LDL-C, low-density lipoprotein cholesterol; H\&E, hematoxylin and eosin; 
EVG, elastica van gieson; IGTT, intravenous glucose tolerance tests; IITT, intravenous insulin tolerance test; AUC, area under carve.

\section{Disclosure of conflict of interest}

None

\section{Acknowledgements}

This study was supported by the National Natural Science of China (No. 81400328 and 81773795) and China Postdoctoral Science Foundation (No. 2015M582800 and 2016T90972) and Natural Science Basic Research Plan in Shaanxi Province of China (2016JM8025) and Shaanxi Social Development Science and Technology Plan (2016SF-295) and Scientific Research Fund of Shannxi Provincial Education Department (17JS116).

QY, EQL and SWW contribute to experimental design, discussion of result and critical revision of the manuscript. QY and RHL contributes to the design of study and draft the manuscript. YQ, RHL, LJH and GWZ contribute to the conduct of experiments. HG and QP contribute to additional experiment and crucial revises in the manuscript. 


\section{References}

Cefalu, W.T., Wang, Z.Q., Bell-Farrow, A.D., Collins, J., Morgan, T., and Wagner, J.D. 2004. Caloric restriction and cardiovascular aging in cynomolgus monkeys (Macaca fascicularis): metabolic, physiologic, and atherosclerotic measures from a 4-year intervention trial. J. Gerontol. A. Biol. Sci. Med. Sci. 59(10): 1007-14. PMID: 15528768.

Cheng, D., Wang, Y., Gao, S., Wang, X., Sun, W., Bai, L., et al. 2015. Atorvastatin delays the glucose clearance rate in hypercholesterolemic rabbits. Biomed. Pharmacother. 72: 24-9. doi:10.1016/j.biopha.2015.03.007. PMID: 26054671.

Colman, R.J., Anderson, R.M., Johnson, S.C., Kastman, E.K., Kosmatka, K.J., Beasley, T.M., et al. 2009. Caloric restriction delays disease onset and mortality in rhesus monkeys. Science, 325(5937): 201-4. doi:10.1126/science.1173635. PMID: 19590001.

Dolinsky, V.W. and Dyck, J.R. 2011. Calorie restriction and resveratrol in cardiovascular health and disease. Biochim. Biophys. Acta, 1812(11): 1477-89. doi:10.1016/j.bbadis.2011.06.010. PMID: 21749920.

Fan, J., Kitajima, S., Watanabe, T., Xu, J., Zhang, J., Liu, E., et al. 2015. Rabbit models for the study of human atherosclerosis: from pathophysiological mechanisms to translational medicine. Pharmacol. Ther. 146: 104-19. doi:10.1016/j.pharmthera.2014.09.009. PMID: 25277507.

Fontana, L., Meyer, T.E., Klein, S., and Holloszy, J.O. 2004. Long-term calorie restriction is highly effective in reducing the risk for atherosclerosis in humans. Proc. Natl. Acad. Sci. U S A, 101(17): 6659-63. doi:10.1073/pnas.0308291101. PMID: 15096581.

Fukuda, S., Kita, S., Obata, Y., Fujishima, Y., Nagao, H., Masuda, S., et al. 2017. The unique prodomain of T-cadherin plays a key role in adiponectin binding with the essential extracellular 
cadherin repeats 1 and 2. J. Biol. Chem. 292(19): 7840-7849. doi:10.1074/jbc.M117.780734. PMID: 28325833.

Gassmann, M., Grenacher, B., Rohde, B., and Vogel, J. 2009. Quantifying Western blots: pitfalls of densitometry. Electrophoresis, 30(11): 1845-55. doi:10.1002/elps.200800720. PMID: 19517440.

Guo, Z., Mitchell-Raymundo, F., Yang, H., Ikeno, Y., Nelson, J., Diaz, V., et al. 2002. Dietary restriction reduces atherosclerosis and oxidative stress in the aorta of apolipoprotein E-deficient mice. Mech. Ageing Dev. 123(8): 1121-31. doi.org/10.1016/S0047-6374(02)00008-8. PMID: 12044962.

Hursting, S.D., Smith, S.M., Lashinger, L.M., Harvey, A.E., and Perkins, S.N. 2010. Calories and carcinogenesis: lessons learned from 30 years of calorie restriction research. Carcinogenesis, 31(1): 83-9. doi:10.1093/carcin/bgp280. PMID: 19969554.

Khanna, V., Jain, M., Singh, V., Kanshana, J.S., Prakash, P., Barthwal, M.K., et al. 2013. Cholesterol diet withdrawal leads to an initial plaque instability and subsequent regression of accelerated iliac artery atherosclerosis in rabbits. PLoS One, 8(10): e77037. doi:10.1371/journal.pone.0077037. PMID: 24146955.

Koubova, J. and Guarente, L. 2003. How does calorie restriction work? Genes. Dev. 17(3): 313-21. doi:10.1101/gad.1052903. PMID: 12569120.

Lacombe, C., Corraze, G., and Nibbelink, M. 1983. Increases in hyperlipoproteinemia, disturbances in cholesterol metabolism and atherosclerosis induced by dietary restriction in rabbits fed a cholesterol-rich diet. Lipids, 18(4): 306-12. PMID: 6865663.

Laforest, S., Labrecque, J., Michaud, A., Cianflone, K., and Tchernof, A. 2015. Adipocyte size as a determinant of metabolic disease and adipose tissue dysfunction. Crit. Rev. Clin. Lab. Sci. 52(6): 301-13. doi:10.3109/10408363.2015.1041582. PMID: 26292076. 
Millen, B.E., Wolongevicz, D.M., de Jesus, J.M., Nonas, C.A., and Lichtenstein, A.H. 2014. 2013 American Heart Association/American College of Cardiology Guideline on Lifestyle Management to Reduce Cardiovascular Risk: practice opportunities for registered dietitian nutritionists. J. Acad. Nutr. Diet, 114(11): 1723-9. doi:10.1016/j.jand.2014.07.037. PMID: 25439080.

Muzumdar, R., Allison, D.B., Huffman, D.M., Ma, X., Atzmon, G., Einstein, F.H., et al. 2008. Visceral adipose tissue modulates mammalian longevity. Aging Cell, 7(3): 438-40. doi:10.1111/j.1474-9726.2008.00391.x. PMID: 18363902.

Pires, R.C., Souza, E.E., Vanzela, E.C., Ribeiro, R.A., Silva-Santos, J.C., Carneiro, E.M., et al. 2014. Short-term calorie restriction improves glucose homeostasis in old rats: involvement of AMPK. Appl. Physiol. Nutr. Metab. 39(8): 895-901. doi:10.1139/apnm-2013-0520. PMID: 24844367.

Roth, L.W. and Polotsky, A.J. 2012. Can we live longer by eating less? A review of caloric restriction and longevity. Maturitas, 71(4): 315-9. doi:10.1016/j.maturitas.2011.12.017. PMID: 22281163.

Skurk, T., Alberti-Huber, C., Herder, C., and Hauner, H. 2007. Relationship between adipocyte size and adipokine expression and secretion. J. Clin. Endocrinol. Metab. 92(3): 1023-33. doi:10.1210/jc.2006-1055. PMID: 17164304.

Wang, Y., Snel, M., Jonker, J.T., Hammer, S., Lamb, H.J., de Roos, A., et al. 2011. Prolonged caloric restriction in obese patients with type 2 diabetes mellitus decreases plasma CETP and increases apolipoprotein AI levels without improving the cholesterol efflux properties of HDL. Diabetes Care, 34(12): 2576-80. doi:10.2337/dc11-0685. PMID: 21994427.

Waqar, A.B., Koike, T., Yu, Y., Inoue, T., Aoki, T., Liu, E., et al. 2010. High-fat diet without excess calories induces metabolic disorders and enhances atherosclerosis in rabbits. Atherosclerosis, 213(1): 148-55. doi:10.1016/j.atherosclerosis.2010.07.051. PMID: 20832799. 
Yu, Q., Li, Y., Wang, Y., Zhao, S., Yang, P., Chen, Y., et al. 2012a. C-reactive protein levels are associated with the progression of atherosclerotic lesions in rabbits. Histol. Histopathol. 27(4): 529-35. doi:10.14670/HH-27.529. PMID: 22374730.

Yu, Q., Li, Y., Waqar, A.B., Wang, Y., Huang, B., Chen, Y., et al. 2012b. Temporal and quantitative analysis of atherosclerotic lesions in diet-induced hypercholesterolemic rabbits. J. Biomed. Biotechnol. 2012: 506159. doi:10.1155/2012/506159. PMID: 22505812.

Yu, Q., Liu, Z., Waqar, A.B., Ning, B., Yang, X., Shiomi, M., et al. 2014. Effects of antisense oligonucleotides against C-reactive protein on the development of atherosclerosis in WHHL rabbits. Mediators Inflamm. 2014: 979132. doi:10.1155/2014/979132. PMID: 24872601.

Yu, Q., Wang, Y., Yu, Y., Li, Y., Zhao, S., Chen, Y., et al. 2012c. Expression of TRPV1 in rabbits and consuming hot pepper affects its body weight. Mol. Biol. Rep. 39(7): 7583-9. doi:10.1007/s11033-012-1592-1. PMID: 22327653.

Zhang, G., Chen, Y., BilalWaqar, A., Han, L., Jia, M., Xu, C., et al. 2015. Quantitative analysis of rabbit coronary atherosclerosis. Practical techniques utilizing open-source software. Anal. Quant. Cytopathol. Histpathol. 37(2): 115-22. PMID: 26065232. 


\section{Figure Legends}

Fig. 1 A schematic diagram of experimental protocol. 50 Japanese white rabbits were fed by a HCD for 12 weeks to develop mild atherosclerosis. Then, these rabbits were randomly divided into four groups, and one group $(\mathrm{n}=10)$ was sacrificed immediately as the baseline control group and another group was continually fed by HCD in another 16 weeks as a positive control group $(n=10)$. The rest of rabbits were divided into two groups and fed with a normal diet instead of a HCD. Among them, the DR group $(n=15)$ was fed by the dietary restriction and received $60 \%$ of the diet consumption in the group that was fed by ad libitum (AL, $n=15)$.

Fig. 2 Weight of body and internal organs. (A) Body weights of three groups were measured per week $\left({ }^{* * * *} \mathrm{p}<0.0001\right.$ as the AL group versus the HCD group; ${ }^{\#} \mathrm{p}<0.05,{ }^{\# \#} \mathrm{p}<0.01$ and ${ }^{\# \# \#} \mathrm{p}<0.001$ as the DR group versus the HCD group; ${ }^{\&} \mathrm{p}<0.01,{ }^{\& \& \&} \mathrm{p}<0.001$ and ${ }^{\& \& \& \&} \mathrm{p}<0.0001$ as the AL group versus the DR group). (B) The major internal organs weight $\left({ }^{* * * *} \mathrm{p}<0.0001\right)$. Data are expressed as the means \pm SE.

Fig. 3 Weight of adipose tissue, size of adipocytes and plasma adiponectin. (A) Representative graphs of visceral adipose tissue (upper), adipose tissues weights (lower) $\left({ }^{*} \mathrm{p}<0.05,{ }^{* *} \mathrm{p}<0.01,{ }^{* * *} \mathrm{p}<0.001\right.$ and $\left.{ }^{* * * *} \mathrm{p}<0.0001\right)$. (B) Representative micrographs of visceral adipose tissue (upper) (bar=100 $\left.\mu \mathrm{m}\right)$, adipocyte cell size distribution and mean diameter in visceral fat (lower; $\mathrm{n}=10$, respectively; ${ }^{*} \mathrm{p}<0.05$ and ${ }^{* *} \mathrm{p}<0.01$ as the AL group versus the DR group). (C) Immunoblotting analysis and quantification of plasma adiponectin $(n=4)$. Data are expressed as the means \pm SE.

Fig. 4 Glucose metabolism. (A) Plasma glucose. (B) intravenous glucose tolerance test and its AUC $(n=8)$. (C) intravenous insulin tolerance test and its $\operatorname{AUC}(n=8) .{ }^{*} \mathrm{p}<0.05$ and ${ }^{* *} \mathrm{p}<0.01$ as the AL group versus the DR group. Data are expressed as the means $\pm \mathrm{SE}$.

Fig. 5 Plasma insulin and glucagon levels. (A) Plasma insulin and (B) glucagon ( $n=8$, respectively). ${ }^{*} p$ $<0.05$ as the AL group versus the DR group. Data are expressed as the means $\pm \mathrm{SE}$.

Fig. 6 Plasma lipid profiles. (A) Plasma TC in the AL and DR groups ( $\mathrm{n}=15$, respectively). (B) Plasma HDL-C and (C) Plasma TG ( $\mathrm{n}=15$, respectively). ${ }^{*} \mathrm{p}<0.05$ and ${ }^{* *} \mathrm{p}<0.01$ as the AL group versus the 
DR group. Data are expressed as the means $\pm \mathrm{SE}$.

Fig. 7 Gross lesion of atherosclerosis. (A) Representative graphs of aortic atherosclerosis stained by Sudan IV (left) and the area of gross lesions were quantified (right; $n=10$ in the baseline group, $n=7$ in HCD group, $n=14$ in the AL group and $n=15$ in the DR group). ${ }^{* *} \mathrm{p}<0.01$ and ${ }^{* * *} \mathrm{p}<0.001$. Data are expressed as the means $\pm \mathrm{SE}$.

Fig. 8 Intimal lesions and components. Serial paraffin cross sections of aortic lesions were stained with H\&E, EVG and immunohistochemically stained with mAbs against either macrophages (MФ) or $\boldsymbol{\alpha}$-smooth muscle actin for SMCs (left), intimal lesions and positively stained areas of immunostained macrophages and SMCs are quantified (right; $\mathrm{n}=10$, respectively) (bar $=200 \mu \mathrm{m}) .{ }^{*} \mathrm{p}<0.05$ and ${ }^{* *} \mathrm{p}<$ 0.01 as the AL group versus the DR group. Data are expressed as the means $\pm \mathrm{SE}$. 


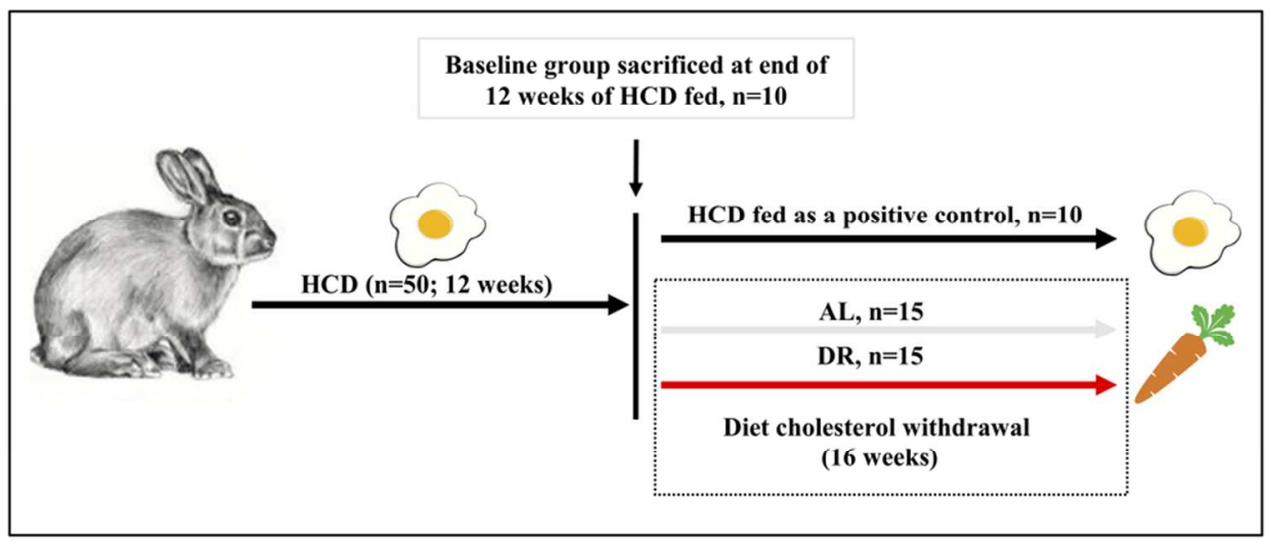

Fig. $1 \mathrm{~A}$ schematic diagram of experimental protocol. 50 Japanese white rabbits were fed by a HCD for 12 weeks to develop mild atherosclerosis. Then, these rabbits were randomly divided into four groups, and one group $(n=10)$ was sacrificed immediately as the baseline control group and another group was continually fed by HCD in another 16 weeks as a positive control group $(n=10)$. The rest of rabbits were divided into two groups and fed with a normal diet instead of a HCD. Among them, the DR group $(n=15)$ was fed by the dietary restriction and received $60 \%$ of the diet consumption in the group that was fed by ad libitum (AL, $\mathrm{n}=15)$.

$85 \times 38 \mathrm{~mm}(300 \times 300 \mathrm{DPI})$ 

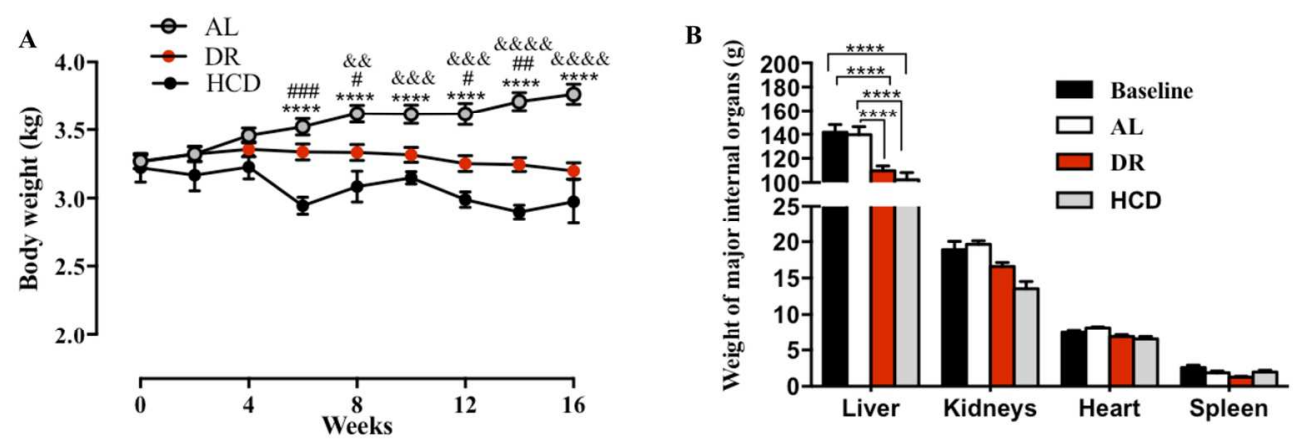

Fig. 2 Weight of body and internal organs. (A) Body weights of three groups were measured per week $(* * * * \mathrm{p}<0.0001$ as the AL group versus the HCD group; \# $\mathrm{p}<0.05, \# \# \mathrm{p}<0.01$ and \#\#\#p<0.001 as the DR group versus the HCD group; \&\&p $<0.01, \& \& \& p<0.001$ and $\& \& \& \& p<0.0001$ as the AL group versus the DR group). (B) The major internal organs weight $(* * * * p<0.0001)$. Data are expressed as the means \pm SE.

$69 \times 23 \mathrm{~mm}(600 \times 600 \mathrm{DPI})$ 

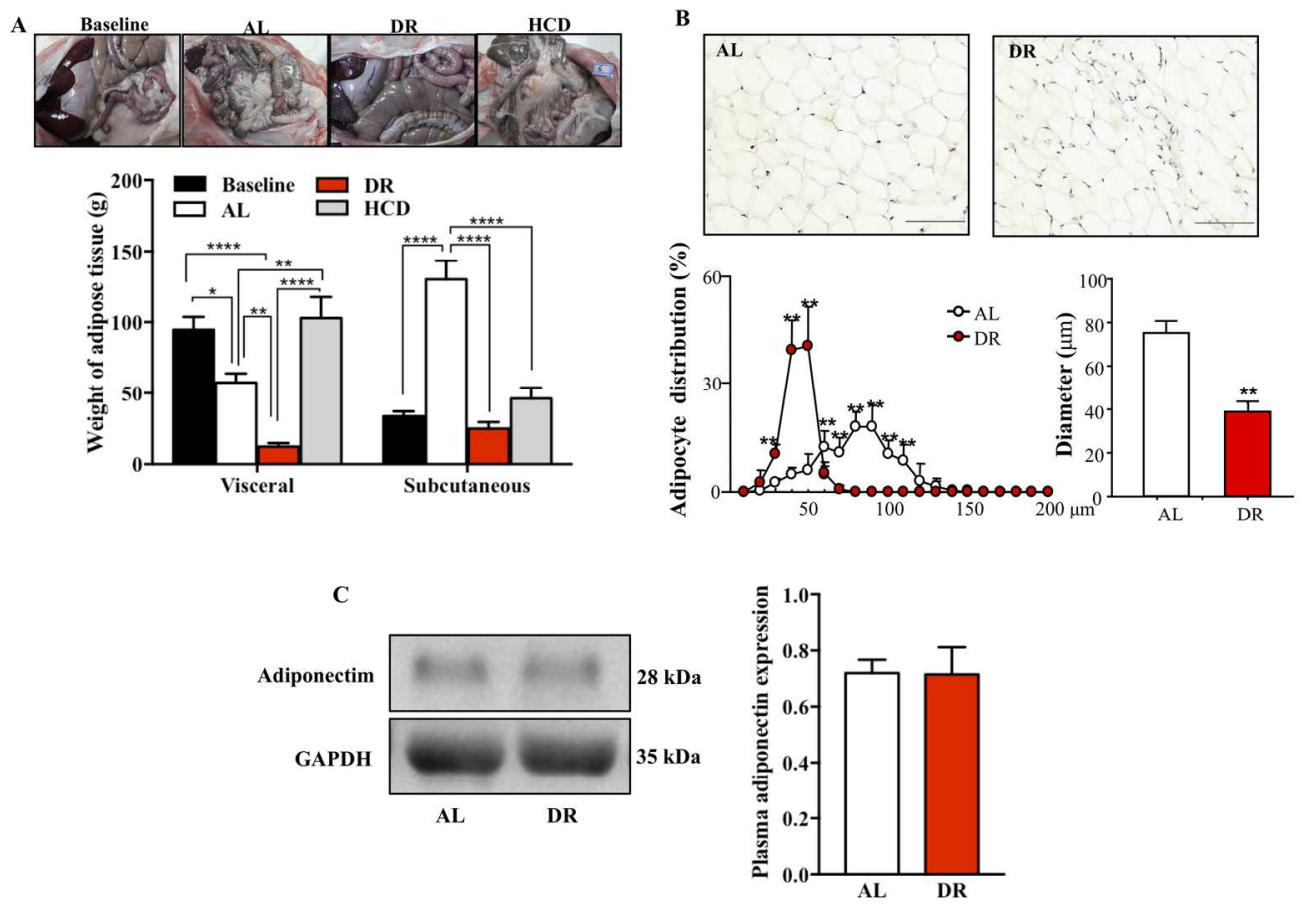

Fig. 3 Weight of adipose tissue, size of adipocytes and plasma adiponectin. (A) Representative graphs of visceral adipose tissue (upper), adipose tissues weights (lower) (* $\mathrm{p}<0.05, * * \mathrm{p}<0.01, * * * \mathrm{p}<0.001$ and $* * * * p<0.0001)$. (B) Representative micrographs of visceral adipose tissue (upper) (bar $=100 \mu \mathrm{m})$, adipocyte cell size distribution and mean diameter in visceral fat (lower; $n=10$, respectively; $* p<0.05$ and $* * p<0.01$ as the AL group versus the DR group). (C) Immunoblotting analysis and quantification of plasma adiponectin $(n=4)$. Data are expressed as the means $\pm S E$.

$163 \times 113 \mathrm{~mm}(300 \times 300 \mathrm{DPI})$ 
A

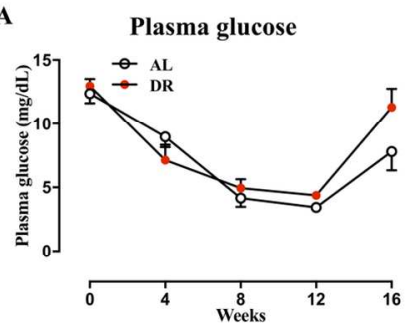

B

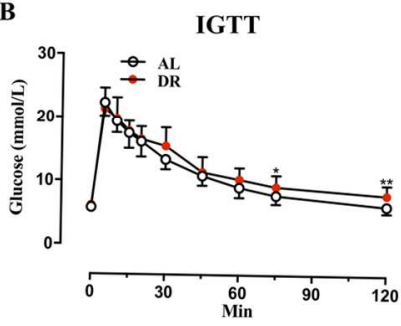

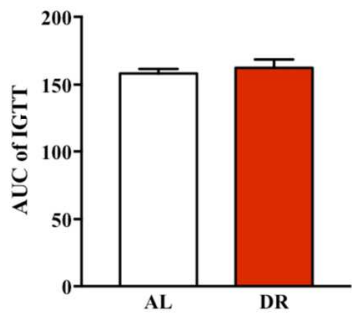

C
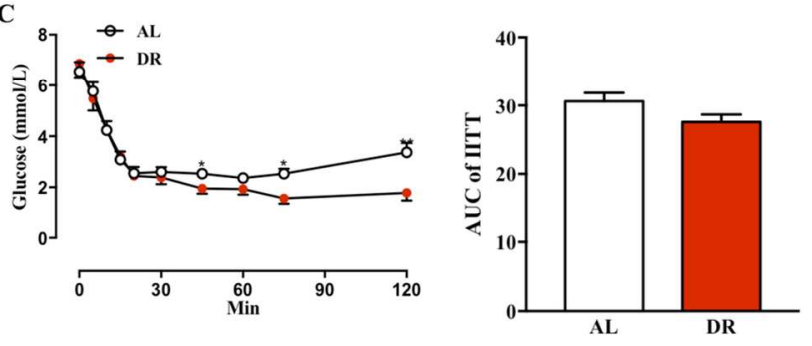

Fig. 4 Glucose metabolism. (A) Plasma glucose. (B) intravenous glucose tolerance test and its $A U C$ ( $n=8$ ). (C) intravenous insulin tolerance test and its AUC $(n=8)$. $* p<0.05$ and $* * p<0.01$ as the AL group versus the DR group. Data are expressed as the means $\pm \mathrm{SE}$.

$$
130 \times 80 \mathrm{~mm}(300 \times 300 \mathrm{DPI})
$$


A

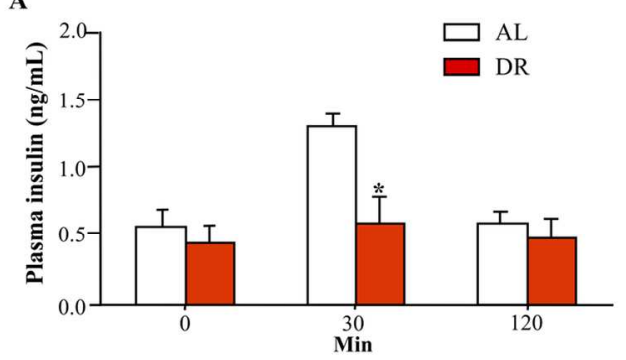

B

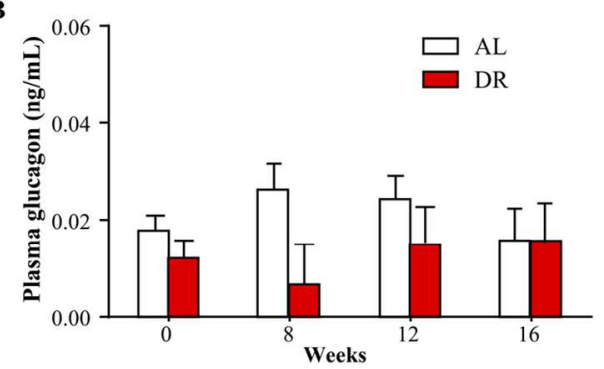

Fig. 5 Plasma insulin and glucagon levels. (A) Plasma insulin and (B) glucagon ( $n=8$, respectively). ${ }^{*} p<$ 0.05 as the AL group versus the DR group. Data are expressed as the means $\pm \mathrm{SE}$.

$64 \times 19 \mathrm{~mm}(600 \times 600 \mathrm{DPI})$ 

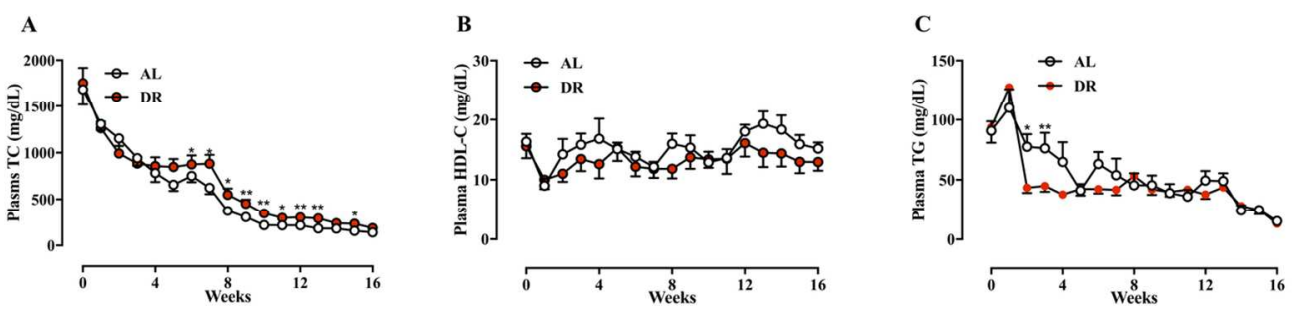

Fig. 6 Plasma lipid profiles. (A) Plasma TC in the AL and DR groups ( $n=15$, respectively). (B) Plasma HDL-C and (C) Plasma TG ( $n=15$, respectively). $* p<0.05$ and $* * p<0.01$ as the AL group versus the DR group. Data are expressed as the means $\pm \mathrm{SE}$.

$60 \times 15 \mathrm{~mm}(600 \times 600 \mathrm{DPI})$ 

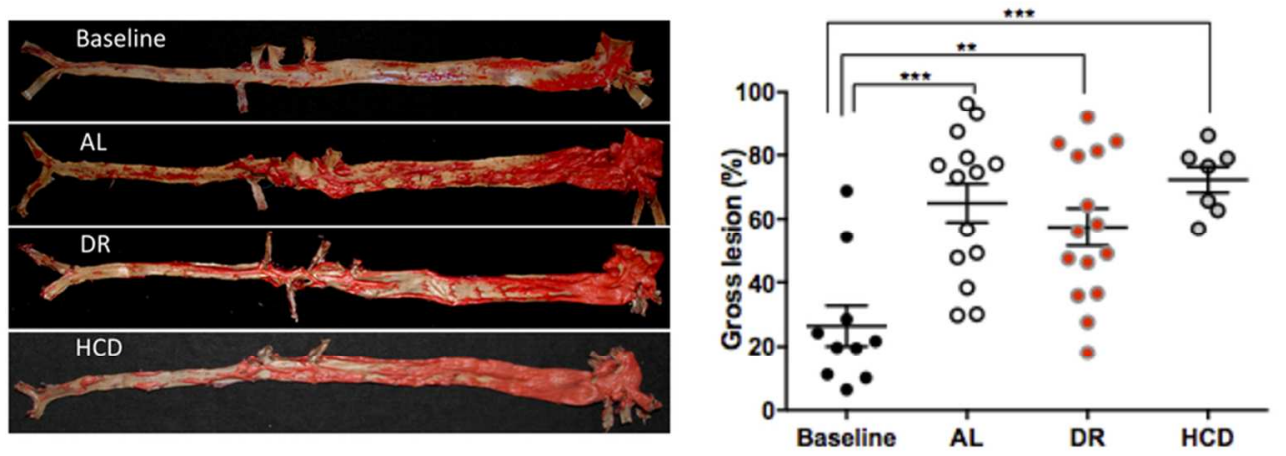

Fig. 7 Gross lesion of atherosclerosis. (A) Representative graphs of aortic atherosclerosis stained by Sudan IV (left) and the area of gross lesions were quantified (right; $n=10$ in the baseline group, $n=7$ in $H C D$ group, $\mathrm{n}=14$ in the $\mathrm{AL}$ group and $\mathrm{n}=15$ in the DR group). $* * \mathrm{p}<0.01$ and $* * * \mathrm{p}<0.001$. Data are expressed as the means \pm SE.

$77 \times 29 \mathrm{~mm}(300 \times 300$ DPI $)$ 

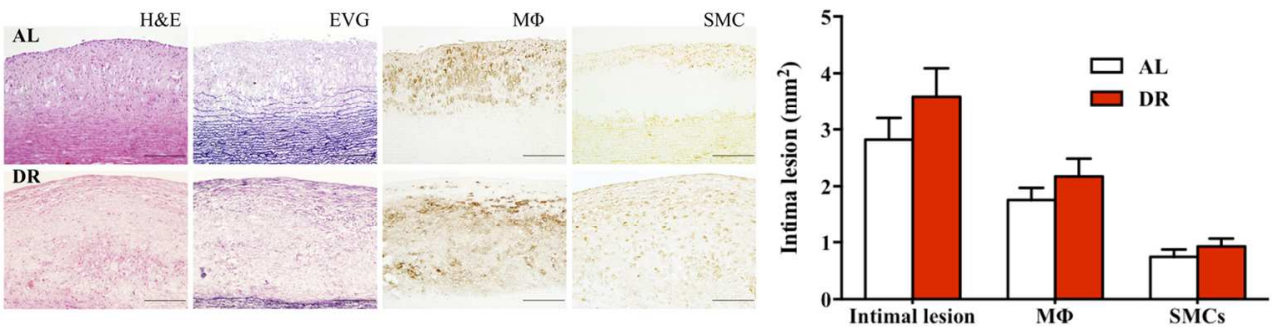

Fig. 8 Intimal lesions and components. Serial paraffin cross sections of aortic lesions were stained with H\&E, EVG and immunohistochemically stained with mAbs against either macrophages (MФ) or a-smooth muscle actin for SMCs (left), intimal lesions and positively stained areas of immunostained macrophages and SMCs are quantified (right; $n=10$, respectively) (bar $=200 \mu \mathrm{m}$ ). $* \mathrm{p}<0.05$ and $* * p<0.01$ as the AL group versus the DR group. Data are expressed as the means $\pm \mathrm{SE}$.

$64 \times 17 \mathrm{~mm}(600 \times 600 \mathrm{DPI})$ 